\title{
Editorial
}

\author{
M. N. Hoda ${ }^{1}$
}

Published online: 21 July 2021

(C) Bharati Vidyapeeth's Institute of Computer Applications and Management 2021

Warm greetings to all our readers!!! We hope this year brings relief and progress to humanity. BJIT remains committed to delivering on its challenge of consistently showcasing and disseminating novel researches pertaining to computing applications and capable of altering the quality of human life. It is a matter of great privilege for me to unveil before you the thirty sixth issue i.e. Volume 13 Number 04 of the "International Journal of Information Technology" [An official Journal of Bharati Vidyapeeth's Institute of Computer Applications and Management (BVICAM), New Delhi] with acronym BJIT. From this volume onwards we have increased our frequency to six issues an year. The issue is live on the Springer content platform SpringerLink and available to the prospective readers through Springer CS package globally.

Throughout the world, nations have started recognizing that Information Technology (IT) is now acting as a catalyst in speeding up the economic activities in efficient governance, citizens' empowerment, sustainable development and in improving the quality of human life. Recent advancements in IT have touched almost every conceivable area of human life. Its degree of pervasiveness, in day to day life, is rapidly increasing, every new day. On the backdrop of this, BJIT has accepted the challenge to consistently showcase, disseminate and institutionalize the rapidly changing huge knowledgebase globally, with authenticity and accuracy, having special focus on the new researches pertaining to IT applications for improving the quality of day to day life.

M. N. Hoda

bjit@bvicam.ac.in

1 International Journal of Information Technology (BJIT), New Delhi, India
Volume 13 Number 04 presents a compilation of forty six papers, chosen out of over 400 manuscripts, that span a broad variety of topics from various emerging areas of Information Technology and Computer Science, especially addressing current research problems related to Grid Computing, Intrusion detection system, power optimization, cancer diagnosis, Text Steganography, Cloud Services and Test Case Priortization; to name a few.

After almost over an year, our world still continues to battle the COVID. Technology is playing a major role in easing human life in the new norm where personal interaction has taken a backseat. The first manuscript in this issue "Modeling mobile apps user behavior using Bayesian networks", Isuru Dharmasena et al. evaluates a strategy to build Bayesian networks and further improve the causal networks using re-sampling methods. Authorizations and signature verifications are important in the real world. The second manuscript "Signature warping and greedy approach based offline signature verification", Abhiram Natarajan et al. propositions a design specifically to generate a dataset from a very minimalistic number of original signatures. As theCOVID-19 pandemic does not seem to offer any immediate relief to humanity in the near future. The next manuscript "Deep-LSTM ensemble framework to forecast Covid-19: an insight to the global pandemic", Sourabh Shastri et al. outlines a nested ensemble model using deep learning methods based on long short term memory (LSTM) to identify and predict COVID-19. The manuscript "An efficient task mapping algorithm for osmotic computing-based ecosystem", Benazir Neha et al. implements a task mapping algorithm by incorporating osmotic computing principles to complete the tasks in latency-sensitive systems. Reliability is an important characteristic of all software systems. The manuscript "Deriving and validating fault metric for 
object oriented systems using use case diagram", Sangeeta Sabharwal et al. proposes a fault metric called use case fault metric (UCFM) for object-oriented systems using use case diagram and is built on the theory of Fault Tree Analysis (FTA). The next manuscript "Enhanced surface passivation with TIPS pentacene and additional interfacial layer for MIS solar cells", Sukriti Kaulgud et al. intends an enhanced surface passivation for MIS solar cells. Multi-objective route optimization is a challenging problem. The manuscript "Hierarchical Bayesian approach for improving weights for solving multi-objective route optimization problem", Romit S. Beed et al. designs a novel Hierarchical Bayesian model based on multinomial distribution and Dirichlet prior to refine the weights for solving such multi-objective route optimization problems. The next manuscript "COVID-19 compliant and cost effective teaching model for King Abdulaziz University", Hisham Bardesi et al. advises a cost-effective hybrid teaching model (CeHTM) for the King Abdulaziz University during the pandemic times. The manuscript "A computational study of B-cell epitopes of wheat allergens and identification of its IgE binding residues", Amogh Johri et al. addresses the identification of IgE binding allergy causing B-Cell epitopes of wheat (Triticum aestivum) allergens. Efficacy of content-based image classification is dependent on the richness of the feature vectors extracted from the image data. The manuscript "Hybrid descriptor definition for content based image classification using fusion of handcrafted features to convolutional neural network features", Rik Das et al. captures the probability distribution of input images to a pre trained Convolution Neural Network by utilizing it as a fixed weight feature extractor. The manuscript "A new parametric generalized exponential entropy measure on intuitionistic vague sets", Taruna et al. suggests a new measure of the exponential theorem on an intuitive set of equations. The manuscript "Configurable memory designs in quantum-dot cellular automata", Mrinal Goswami et al. delineates configurable memory structures are investigated in QCA. Novel technological tools have made dynamic alterations in the way we interact and learn in this environment. The manuscript "Data driven 3D channel estimation for massive MIMO", C. Ismayil Sayed et al. propagates the application of data-driven techniques for 3D channel estimation of massive MIMO. Pitch is an important component of human speech and is the basis for many applications. The manuscript "Novel model for pitch estimation using hybrid DWT-DCT HPS", Dipti Kalra captures a novel, hybrid Discrete Wavelet Transform-Discrete Cosine Transform (DWTDCT HPS) based pitch extraction technique. The manuscript "A hybrid multi-criteria decision making algorithm for cloudservice selection", Munmun Saha et al. offers a hybrid multi-criteria decision-making (H-MCDM) algorithm to find a solution by considering different conflicting QoS criteria. The manuscript "A diagnostic prediction model for colorectal cancer in elderlies via internet of medical things", Parvaneh Asghari et al. details an IoT-based predicting model to predict colorectal cancer (CRC) in elderlies. The manuscript "Automatic handover execution technique using machine learning algorithm for heterogeneous wireless networks", Nishatbanu Nayakwadi et al. presents an efficient, automatic handover mechanism between LTE and mmWave. Agriculture has been an important activity of human civilization since times immemorial. The manuscript "Estimation of crop production using machine learning techniques: a case study of J\&K”, Jyoti Mahajan et al. details a crop yield prediction system using machine learning algorithms (decision trees, random forest, support vector regressor, gradient boosting) which can be used to predict the yields of major crops produced in regions of Jammu and Kashmir in India. Patient centric social networks may help patients suffering from similar diseases communicate with each other. The manuscript "A similarity matrix based approach for building patient centric social networks", S. Bissoyi et al. develops a recommendation strategy for a group of patients in a social network, by deriving similarities in the unstructured clinical text found in their profiles. The manuscript "Speckle noise reduction and entropy minimization approach for medical images", Neha Mehta et al. suggests a novel approach for speckle noise reduction and entropy minimization.

The manuscript, "A novel audio encryption method using Henon-Tent chaotic pseudo random number sequence", Subhajit Adhikari et al. evaluates a novel audio encryption method to provide information security. Modeling student learning behavior is crucial to assess their performance. The manuscript, "A stacked auto-encoder with scaled conjugate gradient algorithm for Malayalam ASR", Leena Pillai et al. investigates a network structured with an unsupervised pre-training followed by supervised training. The manuscript, "ETPAC: ECC based trauma plight access control for healthcare Internet of Things", M. Padmashree et al. details a novel ECC model based Trauma Plight Access Control (ETPAC) to reduce the Shared Secret Key generation time, Encryption, Decryption Time, Cipher-Text size with low Secret Key Size. The manuscript," Web-based Framework for Smart Parking System", Awad Alharbi et al. evaluates a novel IoT based mechanism to reduce the traffic congestion on the streets. The next manuscript, "Comparison and analysis of logistic regression, Naïve Bayes and KNN machine learning algorithms for credit card fraud detection”, Fayaz Itoo et al. empirically analyzes various 
machine learning algorithms for their efficacy in credit card fraud detection. The manuscript, "Development of path planning algorithm for biped robot using combined multi-point RRT and visibility graph", Sarthak Pradhan et al. collates a novel mechanism for solving the path planning problem of the biped robot in a static environment. Varied efforts at applying opinion mining in NLP are being researched. The manuscript," Resource creation for opinion mining: a case study with Marathi movie reviews", N. Mhaske et al. introduces an efficient construction mechanism of opinion corpus and sentiment lexicon that will be used for mining opinions from Marathi language text. The manuscript, "ATSR: distance and time based advanced tour sequence recommendation system", P. S. Singh et al. offers an effective a special type of travel recommendation system that is an advanced tour sequence recommender (ATSR) that outshines the other traditional travel recommendation systems. The manuscript, "Building semantically annotated corpus for text classification of Indian defence news articles", Saurabh Kanekar et al. details a news corpus annotation mechanism and employs an inter-annotator agreement metric to assess the quality of the dataset. The manuscript, "Contextual fully homomorphic encryption schemes-based privacy preserving framework for securing fog-assisted healthcare data exchanging applications", R. Sendhil et al. deploys an efficient contextual fully homomorphic encryption techniques-based privacy preserving framework (CFHET-PPF) for securing fog-assisted health data exchanging applications. The next manuscript, "Sentimental study of CAA by location-based tweets", Geetika Vashisht et al. builds a model to investigate the muchdebated Citizenship Amendment Act (CAA) by analyzing opinionated geo-tagged tweets, manually annotated and cross verified by six annotators. The manuscript, "Design of a flood magnitude prediction model using algorithmic and mathematical approaches", Adannaya Simeon et al. proposes a concise and efficient model that can determine or predict flood and its magnitude using available data from areas prone to flood events. The manuscript, "Fingerprint recognition model using DTCWT algorithm", Pradeep N.R. et al. identifies a novel Dual Tree Complex Wavelet Transformation (DTCWT) fingerprint recognition method for data base FVC 2004 DB 3_A. Efficient mechanisms of ensuring security of mobile devices is becoming all the more important. The manuscript," SonicEvasion: a stealthy ultrasound based invasion using covert communication in smart phones and its security", Ketaki Pattani et al. presents a technical method for depicting unforeseen threats and implementing its security in mobile devices with reference to the novel attack and detection. The manuscript, "Salp swarm bio inspired algorithm for detecting non line of sight vehicles in VANETs", R. Kaviasaran et al. empirically details a Salp swarm bio inspired algorithm for effective localization of the vehicular nodes which is in the NLOS region by utilizing the properties of meta-heuristic approach. Identification of human movement and activity has significant applications in many domains. The manuscript, "Feature fusion using deep learning for smartphone based human activity recognition", Dipanwita Thakur et al. emulates and proposes the fusion of both the handcrafted features and automatically extracted features using deep learning (DL) for HAR model to enhance the performance of HAR. The next manuscript, "Design an optimal digital phase lock loop with current-starved ring VCO using CMOS technology", Rekha Yadav et al. simulates the design of an optimal and low power Digital Phase Lock Loop (DPLL. Deep neural networks are playing a major role in semantic text similarity for natural language processing. The manuscript, "Long short-term memory network for learning sentences similarity using deep contextual embeddings", Suraj Meshram simulates to a deep contextual long semantic textual similarity network. The next manuscript, "Smart transportation system for public universities", Alex Pacheco et al. proposes a mobile application based on Android for the geolocation of buses in Public Universities. HR personnel in organization necessarily need to validate the history of people hired. The manuscript, "Fearless path for human resource personnel's through analytics: a study of recent tools and techniques of human resource analytics and its implication", Mansi Saxena et.al. elaborates the importance and implication of analytics on various HR data and decisions. The manuscript. "Named entity recognition: a semi-supervised learning approach", H. Sintayehu et al. proposes a novel graph-based label propagation algorithm for the Amharic NER problem. The symptoms of disease manifest in the pulse long before the actual disease. The manuscript, "Machine learning based analysis of pulse rate using Panchamahabhutas and Ayurveda", Damini Niranjan et al. offers mechanism to get to the root cause of the disease instead of just treating it based on the visible signs and symptoms. The next manuscript, "Stochastic simulator for priority based task in grid environment", Sunita Rani outlays a probability based scheduling approach for solving the scheduling problem. In the present world online news has become a significant mechanism of immediate news dispersal. The manuscript, “TenzinNet for handwritten Tibetan numeral recognition", Adiyillam Vijaylakshmi et al. develops a convolutional neural networks based architecture named as TenzinNet to recognize handwritten Tibetan numerals. The manuscript, "A novel Hadith authentication mobile system in Arabic to Malay language translation for android and iOS Phones", Alaba 
Fadele et al. lays an efficient mechanism to analyze the key problem of Hadith authentication and scrutiny. The last manuscript, "Design and development of $4 \times 4$ MIMO antennas for smart 5G devices", Vidyadhar Melkeri et al. proposes the design of $5 \mathrm{G}$ Multiple Inputs and Multiple Outputs (MIMO) antenna structure.

I am sure the contributions in this issue, which is an amalgamation of novel trends and technologies to improve our life and sustainability in the present environment, will not only enrich our reader's knowledgebase but will also motivate many of the potential researchers to take up these challenging application areas and contribute effectively for the overall prosperity of the mankind.

As a matter of policy, all the manuscripts received and considered for the Journal, are double blind peer reviewed by at-least two independent referees. Our panel of expert referees posses a sound academic background and have a rich publication record in various prestigious journals representing Universities, Research Laboratories and other Institutions of repute, globally. Finalizing the constitution of the panel of referees, for double blind peer review(s) of the considered manuscripts, was a painstaking process, but it helped us to ensure that only the best, interesting and novel of the considered manuscripts are showcased and that too after undergoing multiple cycles of review, as required.

I wish to express my sincere gratitude to the entire editorial board, members of the resident editorial team and our panel of experts in steering the considered manuscripts through multiple cycles of review and bringing out the best from the contributing authors. I thank my esteemed authors for having shown confidence in BJIT and considering it a platform to showcase and share their original research work. I would also wish to thank the authors whose papers could not have been published in this issue of the Journal, probably because of the minor shortcomings. However, I would like to encourage them to actively contribute for the forthcoming issues.

I will fail in my duty, if I do not thank the members of the team from the Springer, particularly Ms. Suvira Srivastav, Mr. Madan Ellappan, Ms. Jeyapradha Saravanan, Ms. Deepika Sureshkumar and Ms. Nidhi Chandok for their constant support in realizing the issue and presenting it before you.

The undertaken Quality Assurance Process involved a series of well defined activities that, I trust, went a long way in ensuring the quality of the publication. Still, there is always a scope for improvement, and so, I request the contributors and readers to kindly mail me their criticism, suggestions and feedback at bjit@bvicam.ac.in and help in further enhancing the quality of forthcoming issues.

\section{N. Hoda,} Editor-in-Chief, International Journal of Information Technology (BJIT). 